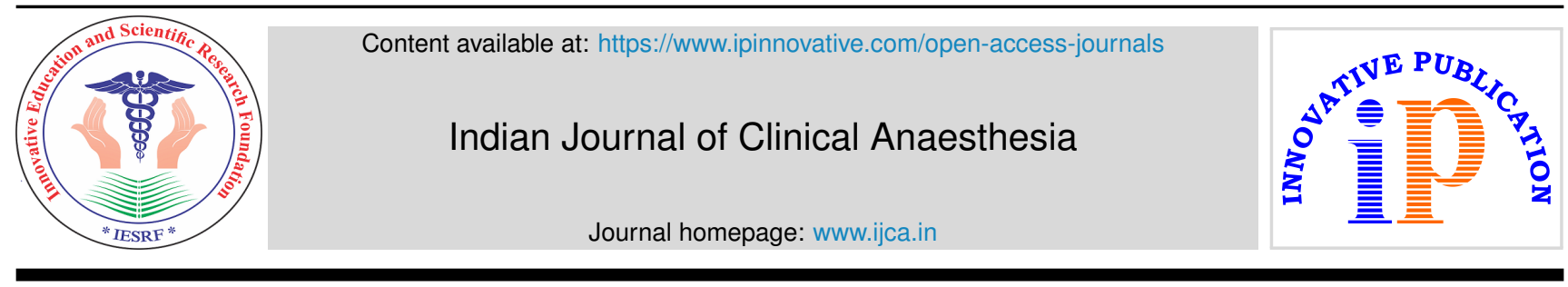

\title{
Editorial
}

\section{Redefining monitoring approaches to minimize organ injury}

\author{
Pallavi Ahluwalia ${ }^{\circledR}$,*, Rachna Wadhwa ${ }^{2}$ \\ ${ }^{1}$ Dept. of Anaesthesia, Teerthanker Mahaveer Medical College, Moradabad, Uttar Pradesh, India \\ ${ }^{2}$ Dept. of Anaesthesiology, G B Pant Hospital, Delhi, India
}

\section{A R T I C L E I N F O}

Article history:

Received 07-02-2021

Accepted 09-02-2021

Available online 10-09-2021

\begin{abstract}
This is an Open Access (OA) journal, and articles are distributed under the terms of the Creative Commons Attribution-NonCommercial-ShareAlike 4.0 License, which allows others to remix, tweak, and build upon the work non-commercially, as long as appropriate credit is given and the new creations are licensed under the identical terms.
\end{abstract}

For reprints contact: reprint@ipinnovative.com
Assessment of organ perfusion at micro-circulatory level is the need of the hour to optimise organ functions. Preservation of organ function depends on a multitude of factors like adequate circulation, oxygen tension in blood, haemoglobin levels, body temperature, nutrition and metabolism. Apart from standard ASA monitoring, there exist paraphernalia of monitors to assess specific functions in a particular organ. These monitors may be noninvasive or invasive. Firstly, cerebral haemodynamic and oxygenation can be monitored by Trans cranial Doppler (TCD), Bi spectral Index (BIS), Near-infrared spectroscopy (NIRS), jugular oximetry and retinal oximetry. Continuous monitoring is helpful in detecting early ischaemia and prevention of stroke and neuro-cognitive dysfunction.

For assessment of cardiac functions, commonly used monitors include invasive blood pressure monitoring, transthoracic echocardiography, trans-esophageal echocardiography, non-invasive cardiac output monitors and pulmonary artery (PA) catheters. PA catheters (Swan-Ganz), helps us in providing details like Cardiac Output(CO), RA pressures, PA pressures, PCWP(pulmonary capillary wedge pressure), Left ventricular end diastolic pressure (LVEDP), mixed venous oxygen saturation, systemic vascular resistance and pulmonary vascular resistance. Maintaining haemoglobin levels more than $10 \mathrm{gm} \%$ is also important for oxygen delivery. However, the precarious balance

\footnotetext{
* Corresponding author.

E-mail address: pallaviahluwalia22@gmail.com (P. Ahluwalia).
}

between the delivery of oxygen (DO2) and consumption of oxygen (VO2) determines optimal tissue perfusion and the prevention of dysfunction of organs. As the major factor determining DO2 is cardiac output, patients' assessment and various resuscitative strategies revolve around monitoring and optimization of this parameter. The precise estimation of $\mathrm{CO}$ can be measured by invasive (Swan Ganz Catheter), minimally invasive, or non-invasive cardiac output monitors. Clinically, there are wide variations in equipment and technologies used in current practice; the definitive answer to which hemodynamics determine the outcome remains elusive. Perioperative goal directed therapy (GDT) refers to an objective system of protocols to standardize the targets and is aimed at optimizing hemodynamics, including the delivery of oxygen to tissues and organ perfusion pressure. It helps a clinician in guiding the appropriate choice, dose and use of vasopressors, inotropes, or the need for fluid therapy. Literature search reveals numerous RCTs comparing perioperative goal-oriented hemodynamic therapy (GDT) with the usual standard of care in various areas of perioperative medicine. There is substantial evidence that perioperative GDT helps in improving perioperative outcomes in patients undergoing surgery. ${ }^{1,2}$ However, despite the evidence, perioperative GDT is still practiced poorly in routine clinical scenario, even in high-resource settings. Perioperative therapeutic interventions are often subjective rather than structured treatment algorithms based on advanced hemodynamic 
monitoring. ${ }^{3,4}$

Saugel et $\mathrm{al}^{5}$ proposed the ' 5 Ts' to specifically describe the concept and framework of the perioperative GDT: target population, intervention timing, types of intervention, target variable, and target value. ${ }^{5}$ They pointed that identification of high-risk patient prior to surgery is prudent to reduce perioperative morbidity and mortality. Therefore, to ensure that perioperative GDT will benefit a patient depends a lot on pre-operative risk stratification. Perioperative GDT can improve postoperative outcomes in high risk patient. Prompt initiation, using different interventions such as fluids, vasopressors, and inotropes and targeting protocols plays a crucial role. Lastly, tailored target values for haemodynamic variables should be used according to the patient's cardiovascular status, dynamic haemodynamic monitoring, personalised targets, and multiple parameters, including physiological targets. Another recent study compared the cardiac output measured by MAP with other variables measuring tissue perfusion such as PPV, Cardiac index, etc., and concluded that microcirculatory perfusion is a real indicator of tissue perfusion. Cell dysfunction is a basic component of shock definition even in the absence of hypotension. Tissue oxygenation refers to the net product of the delivery and the consumption of oxygen at micro-circulatory level. Delivery of oxygen is calculated by formula; $\mathrm{DO} 2=$ Cardiac output $\mathrm{x} 1.39 * \mathrm{Hb} \times \mathrm{SaO} 2$. Tissue perfusion monitoring includes biomarkers of global tissue perfusion and measurements of perfusion in nonvital organs. The tissue metabolism assessment include mixed venous oxygen saturation $(\mathrm{SvO} 2)$ measured by the pulmonary artery catheter and its surrogate central venous oxygen saturation $(\mathrm{ScvO} 2)$ measured from the superior vena cava. The most commonly used parameters are the assessment of global oxygen extraction (VO2/DO2). Hemoglobin, arterial oxygen saturation, $\mathrm{CO}$, and oxygen consumption are the main factors that influence ScvO2. Practically, various physiological, pathophysiological, and therapeutic factors affect venous oxygen saturation such as anemia, hypovolemia, contractility; bleeding, sedation, pain and fever. ${ }^{6}$ Global perfusion can be assessed by serum lactate levels, mixed and central venous oxygen saturation, CO2 gap, and left ventricular strain. Local perfusion markers include skin temperature, central-to-toe temperature difference, skin mottling, capillary refill time, peripheral perfusion index, and tissue oxygen saturation (StO2).

In conclusion, the preservation of organ function is a multimodal physiological approach. Three parameters, such as local tissue perfusion, oxygenation and driving pressure, influence organ function, and targeting these would certainly help to prevent organ injury and significantly reduce mortality. A clear understanding of the $5 \mathrm{~T}$ 's is essential to improve patient outcomes. Monitoring of tissue perfusion is a key to prevent organ dysfunction.

\section{Conflict of Interest}

None.

\section{References}

1. Cecconi M, Corredor C, Arulkumaran N, Abuella G, Ball J, Grounds RM, et al. Clinical review: Goal-directed therapy-what is the evidence in surgical patients? The effect on different risk groups. Crit Care. 2013;17(2):209.

2. Chong MA, Wang Y, Berbenetz NM, Mcconachie I. Does goal-directed haemodynamic and fluid therapy improve peri-operative outcomes? A systematic review and meta-analysis. Eur J Anaesthesiol. 2018;35:46983.

3. Cannesson M, Pestel G, Ricks C, Hoeft A, Perel A. Hemodynamic monitoring and management in patients undergoing high-risk surgery: a survey among North American and European anesthesiologists. Crit Care. 2011;15:R197.

4. Pearse RM, Ackland GL. Perioperative fluid therapy. BMJ. 2012;344:e2865.

5. Saugel B, Kouz K, Scheeren TWL. The ' 5 Ts' of perioperative goaldirected haemodynamic therapy. Br J Anaesth. 2019;123(2):103-7. doi:10.1016/j.bja.2019.04.048.

6. Beest P, Wietasch G, Scheeren T. Clinical review: use of venous oxygen saturations as a goal - a yet unfinished puzzle. Crit Care. 2011;15:232.

\section{Author biography}

Pallavi Ahluwalia, Professor (D) https://orcid.org/0000-0001-8730-3730

Rachna Wadhwa, Associate Professor

Cite this article: Ahluwalia P, Wadhwa R. Redefining monitoring approaches to minimize organ injury. Indian J Clin Anaesth 2021;8(3):352-353. 